\title{
Satellite Image Classification and Content-Base Image Retrieval Using Type-2 Fuzzy Logic
}

\author{
Prof. Anita Thengade ${ }^{1}$, Shirish A. Agale ${ }^{2}$ \\ ${ }^{1}$ Assistant Professor, Computer Engineering, MIT COE, Kothrud, Pune, India \\ ${ }^{2}$ Computer Engineering, MIT COE, Kothrud, Pune, India
}

\begin{abstract}
The detection of oceanic structures, such as upwelling's or eddies, from satellite images has significance for marine environmental studies, coastal resource management, and ocean dynamics studies. There is a lack of tools that allow us to retrieve automatically relevant structures from large satellite image databases. This Paper focuses on the development and validation of a Content-Based Image Retrieval (CBIR) system to classify and retrieve oceanic structures from satellite images with improved accuracy. The image we obtain from http://oceancolor.gsfc.nasa.gov/, which give images from different satellite like Sea-viewing Wide Field-of-view Sensor (SeaWiFS), Moderate Resolution Imaging Spectroradiometer (MODIS), or MEdium Resolution Imaging Spectrometer (MERIS). During the processing of satellite images, different types of imperfection (uncertainties, ambiguities and vagueness) can occur. These imperfections can involve the gray-level intensities, the geometrical characteristics and relations among image objects, and the expert knowledge itself used to overcome these problems. The CBIR system includes several types of soft computing technologies, such as neural networks and fuzzy logic. An advantage of fuzzy logic approach is that the system can be more comprehensible to human users because fuzzy databases (DBs) manage a terminology close to natural languages. This system give retrieval of most relevant and typical oceanic structures, such as upwelling, eddies and wakes.
\end{abstract}

Keywords: Image Classification, Fuzzy Logic, Content-Base Image Retrieval, Fuzzy Database

\section{Introduction}

The use of satellite imagery in everyday life is by no means a novelty. Since the first satellites dedicated to imaging land areas were placed in orbit in 1972 (Landsats), weather reports have become about the most recognizable by-product of humanity's optical dominance of the earth's atmosphere. The excitement and curiosity of viewing our planet from afar has recently led to propositions of sending satellites far into stellar orbit simply to have a live image of our planet. Anyone with a personal computer and modem can, in minutes, have a near-instant satellite image of almost any part of the globe. The rapid advances and accessibility of computer technology has placed satellite technology in millions of homes, cars, schools, and offices. The potential practical use of this technology by amateur and other non-technically expert institutions and individuals is only now beginning to surface.

Satellite images have many applications in meteorology, oceanography, fishing, agriculture, biodiversity conservation, forestry, landscape, geology, cartography, regional planning, education, intelligence and warfare. Images can be in visible colors and in other spectra. There are also elevation maps, usually made by radar images. Interpretation and analysis of satellite imagery is conducted using specialized remote sensing applications.

Satellite image processing plays a vital role for research and developments in "remote sensing", GIS, "agriculture monitoring", disaster management and many other fields of study. However, processing these satellite images requires a large amount of computation time due to its complex and lengthy processing criteria. The most common barrier in an image derived from an imaging device is its imperfection. The acquired image can be inconsistent, incomplete, uncertain or a completely a-miss. All these seems to be the main barrier in real time decision making but to switch the job faster, fuzzy has proved to be an efficient solution. Since, the main problem lies in providing a better and reliable technique which can provide high performance for digital image analysis (even in situations with uncertainty in Gray level, texture, contours, edges detection, relationship between two segments of an image and all other noisy input conditions), with maximum efficiency and minimum manpower utilization. Fuzzy is one such technology that can implement this with ease and in much less time by classifying the image with a procedure, which automatically categorizes all the pixels of image into 


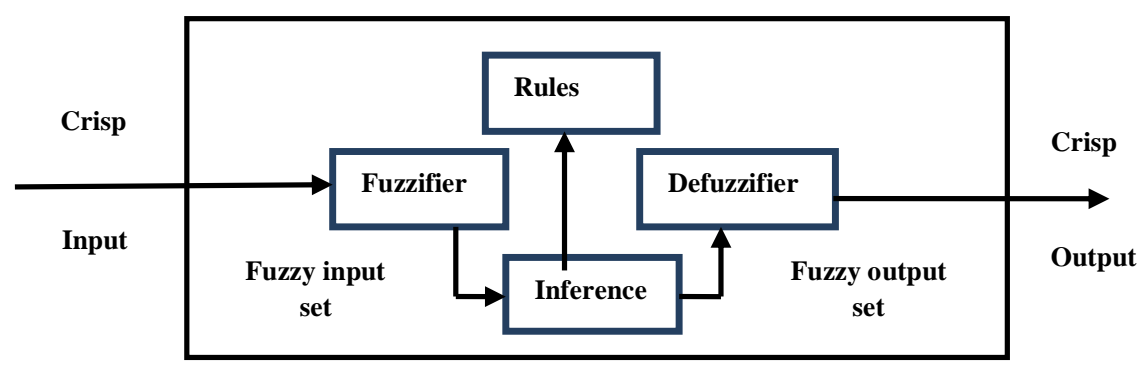

Fig.1. Proposed System

land cover classes and other possible themes. The general architecture of a fuzzy logic system (FLS) which consists of four important components: fuzzifier, rules, inference engine, and defuzzifier are shown in Fig. 1.

The fuzzifier transforms the crisp set values to fuzzy sets by applying fuzzification function. The rules and inference engine are the main component of fuzzy logic system which simulates the human reasoning process by making fuzzy inference on the inputs with IF THEN rules. If we consider the satellite images, input data is not in the form of true color image but for demonstration purpose, a three band $\{\mathrm{R}$ (red) $\mathrm{G}$ (green) $\mathrm{B}$ (blue) $\}$ true color image was taken. Each pixel has a particular color, color being described by the amount of red, green and blue components in it.

Defuzzifier converts the resultant fuzzy set back to a crisp value set which is the system output. Generally, rules are constructed and the output membership functions are arranged in random order without considering the effect of their position on the output, which leads to decline in accuracy of classification. However, if the arrangements of membership functions in output are carefully selected, it leads to a tremendous rise in accuracy. A better arrangement will be, to put membership functions in the output, adjacent to each other if their input membership functions are close or overlapping. The concept was implemented on multi spectral data lines with the spectral pattern (set of radiance measurement obtained in the various wavelength bands for each pixel) used as the numerical basis for categorization using the notion of the normalized fuzzy matrices. This can be implemented with Mamdani-type or Sugeno-type fuzzy inference techniques (Mamdani we are using in this project). The method has been implemented by incorporating the suggested fuzzy logic-based representations with assumptions that the fuzziness of all the optimization formulation parameters are true and only spectral and radiometric characteristic of image pixels being considered without using any geometrical and topological relation between the pixels. Finally, changes were made in the arrangement and type of membership function to analyze the variable effects of these changes on the output. The results obtained clearly demonstrate the consistency and robustness of the developed approach.

\section{Fuzzy Model}

A fuzzy control action consists of situation and action pairs. Conditional rules described in IF and THEN statements are generally used. The IF portion is the ANTECEDENT and the THEN portion is the consequent.

In a fuzzy inference system, the $\mathrm{i}^{\text {th }}$ rule can be described using the first-order as follows:

If $\mathrm{X}_{1}$ is $\mathrm{A}_{\mathrm{i} 1} \mathrm{AND} \ldots$. AND $\mathrm{X}_{\mathrm{L}}$ is $\mathrm{A}_{\mathrm{iL}}$, then $\mu_{\mathrm{i}}$ if $f_{\mathrm{i}}\left(\mathrm{X}_{1} \ldots \mathrm{X}_{\mathrm{L}}\right)$

Where,

$\mathrm{X}_{1} \ldots \mathrm{X}_{\mathrm{L}} \quad$ input variables to fuzzy inference system;

$A_{i 1} \ldots \ldots A_{i L} \quad$ membership function of each input variable for $i^{\text {th }}$ rule;

$\mu_{i} \quad$ output of $i^{\text {th }}$ rule;

$q_{\mathrm{ij}} \quad$ weighting value of $\mathrm{j}^{\text {th }}$ input onto the $\mathrm{i}^{\text {th }}$ output;

$\mathrm{n} \quad$ number of rules;

$\mathrm{L} \quad$ number of input variables;

fi $\left(\mathrm{X}_{1}, \ldots ., \mathrm{X}_{\mathrm{L}}\right)$ real value of consequent part.

There is no restriction on the shape of a membership function. Generally, the triangular, bell-shaped, or Gaussian functions are usually adopted in the formula of the membership function.

\section{Literature Review And Motivation}

Interval type-2 fuzzy systems can be of great help in image analysis and pattern recognition applications. In particular, edge detection is a process usually applied to image sets before the training phase in recognition systems. There are two edge detection technique explained in this paper [1], Sobel and 
Morphological Gradient. According to results shown in paper edge detection technique applied on ORL face database give information that Morphological Gradient gives better results than Sobel.

Pattern recognition and automatic image understanding in remote sensing are important fields to manage the large volume of data acquired from the space during the last 40 years. Paper describes oceanic Remote sensing i.e. oceanic satellite image classification and content-based image retrieval. Paper [2] gives result on NeuroFuzzy classifier. It gives information that with less number of Membership functions and Rules accuracy rate can be high.

The paper [3] presents an edge detection method based on the morphological gradient technique and generalized type-2 fuzzy logic. The theory of alpha planes is used to implement generalized type-2 fuzzy logic for edge detection. For the defuzzification process, the heights and approximation methods are used. When comparing the defuzzification methods used in the generalized type-2 fuzzy inference systems, the heights method achieved better results in image without noise, and the method of approximations in images with noise.

During the processing of satellite images, we encounter different types of imperfection (uncertainties, ambiguities and vagueness). These imperfections can involve the gray-level intensities, the geometrical characteristics and relations among image objects, and the expert knowledge itself used to overcome these problems. If one tries to catch these imperfections by crisp formulations of the processing steps, one may lose valuable information, which could possibly facilitate the next phases of processing. Fuzzy sets in general, and fuzzy image processing in particular can assist us in overcoming imperfections in designing image processing algorithms. After successful image processing system give retrieval of most relevant and typical oceanic structures, and also predict conditions such as upwelling, eddies and wake.

\section{Proposed System}

The structure of the system for classification and retrieval of oceanic structure is shown in Fig. 2

- Input: Original Satellite Images from NASA Website.

- Output: Two outputs will generate proposed system: 1) Classification output which gives regions like Upwelling, Eddies or Wakes. 2) Retrieval of Similar regions which present in Database.

- Image Processing: It is used to improve the quality of original satellite image.

- Content Based Image Retrieval: It is used for retrieving similar regions which are present in Fuzzy Database.

- Type-2 Fuzzy Classification: It is used for classification of regions generated in Image Processing module and give one of the output of system(i.e. Upwelling, Eddies or Wakes)

- Fuzzy Database: Two modules are connected to Fuzzy Database those are Content Based Image Retrieval and Type-2 Fuzzy Classification. It gives efficient managing and searching of satellite images because they are organized without redundancies.

\section{Methodology And The Solution}

The fuzzy CBIR system is composed by these three steps.

- Satellite image processing: The most important task used to improve quality of image with the help of increasing contrast level of image.

- Type 2 Classifier: Type 2 classifier has been developed for the classification of oceanic structures.

- Fuzzy Queries: The human can search oceanic structures using language with structure close to natural language.

\section{A. Image Processing:}

The goal of image processing is to improve the quality of image for next steps. Image processing will perform with help of below steps and see Fig. 3

- Input: Original Satellite Images.

- Output: Improved quality Images.

- Cloud Masking: Cloud masking process detects cloud pixel that are present in original satellite image. Cloud regions and areas with very high standard deviation around each pixel are usually considered as clouds and such cloud pixels are rejected in this process.

- Histogram equalization: It is used for increasing contrast in original image. It is used for showing more visual details to the human user in final image.

- Identification of Island-Continent: It means it defines continent like portion of land cut by right edge of image. Islands are identified because those are surrounded by water regions. 


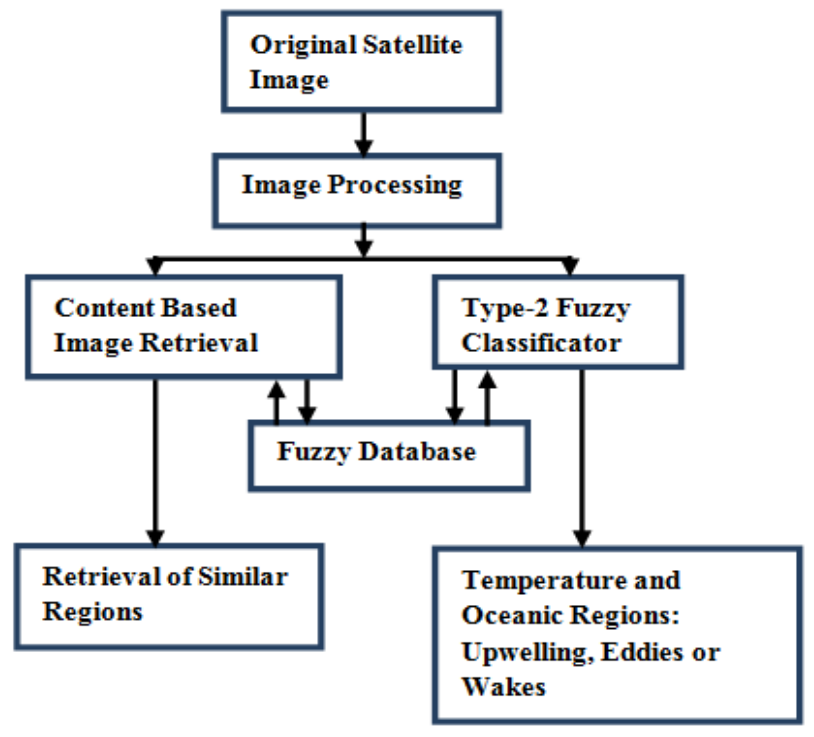

Fig.2. Proposed System

- Delete Regions over a Threshold: Delete regions with area larger or smaller than several preestablished thresholds such regions are called as noninterest regions.

- Edge Detection using Morphological Gradient Type-2 Fuzzy Logic: In morphological gradient, we calculate difference between the intensity values of two neighboring pixels (D) and substitute sum of such D in Equation

$$
\text { MG_edge }=\mathrm{D} 1+\mathrm{D} 2+\mathrm{D} 3+\mathrm{D} 4
$$

\section{B. Type-2 Classification:}

The goal of Type- 2 classification is to find output condition such as upwelling, eddies and wake. Type-

2 classification will perform with help of below steps Fig. 4

- Input: Dataset i.e. improved quality image.

- Output: Oceanic Regions upwelling, eddies or wakes.

- Fuzzifier: Maps crisp input of image into T2FS, which are subsequently used as input to the Type-2 inference engine.

- Sugeno Inference system: It is computationally efficient. It works well with linear techniques (e.g., PID control).It works well with optimization and adaptive techniques. It has guaranteed continuity of the output surface. It is well suited to mathematical analysis.

- Domain Expert Knowledge: It is difficult to acquire good control performance for the system whose scaling factors are just totally obtained from expert's experience.

- Automatic Rule Generation: With help of Domain Expert Knowledge and Karman Filter algorithm we can generate rule automatically [5].

- Karnik-Mendel Type Reducer: It uses new switch point to compute the bounds of type-reduced set It is used to relate input and output of an IT2 Fuzzy controller. Its Performance is high because it uses Footprint of Uncertainty (FOU) [4].

- Defuzzifier: Convert output of inference system into required format.

Some example rules for the classification are shown as follows.

- IF DistToLand is low AND degree of circumscription ellipse is medium-low AND invHu1 is low AND TempDiff is high, THEN is a wake

- IF DistToLand is medium-low AND degree of circumscription ellipse is low AND invHu1 is high AND TempDiff is medium-high, THEN is an upwelling.

\section{Fuzzy Logic for Image Retrieval:}

The retrieval of oceanic structures consists of locating similar regions in the image DB. In this case, the retrieval is carried out by means of the execution of fuzzy queries on the system DB. Automatic image retrieval using fuzzy logic can be described in the following steps: 


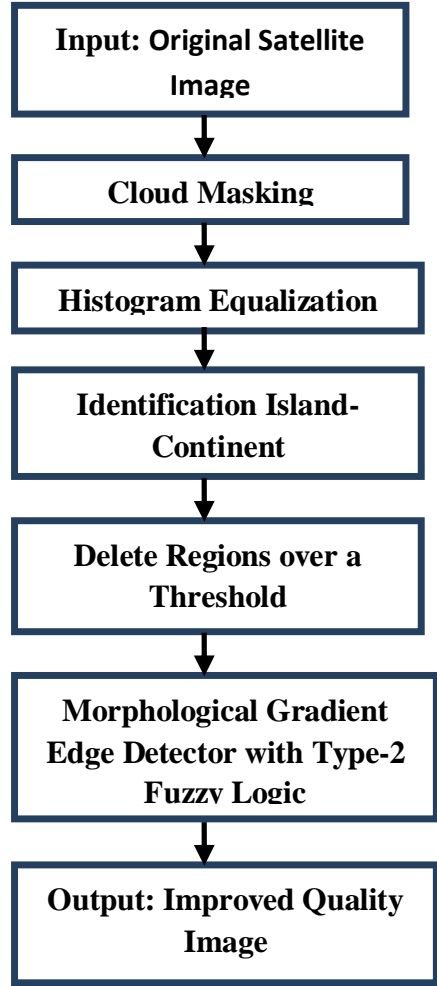

Fig.3. Image processing module

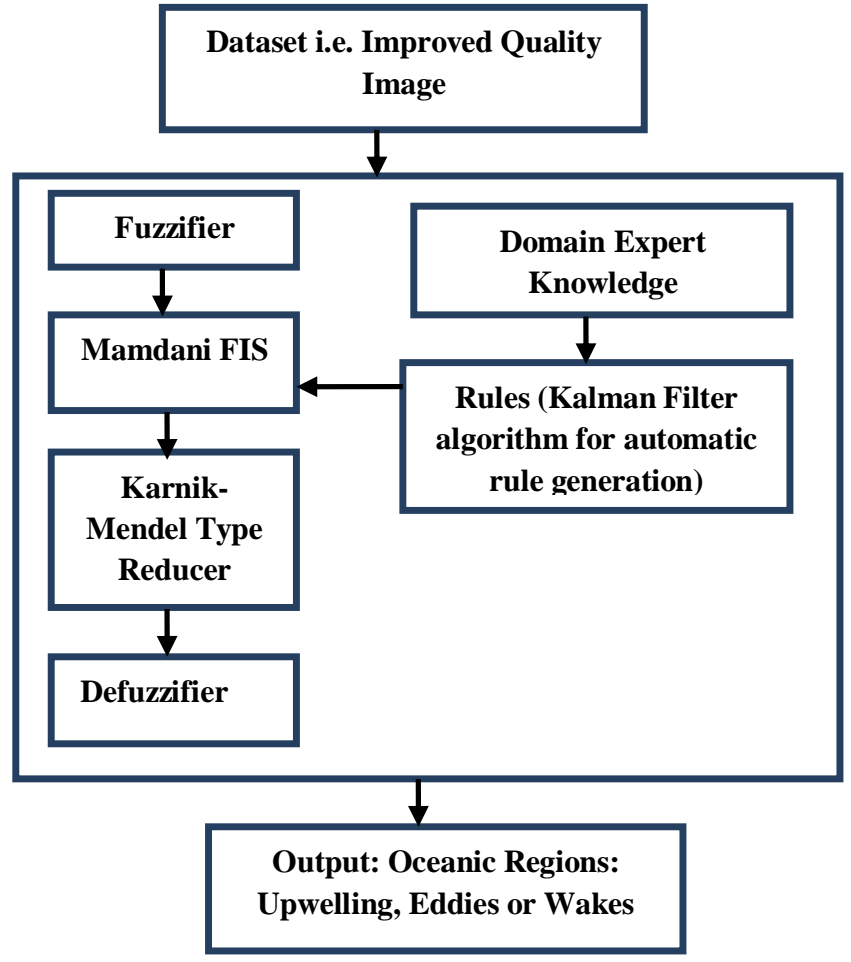

Fig.4. Type-2 Fuzzy Classification Module

- The user has to select a specific oceanic region for retrieving.

- The system searches among all existing regions in the fuzzy DB and selects with a degree of similarity (equal or higher) all similar regions.

\section{Conclusion}

This paper has presented a CBIR system that allows the classification and retrieval of oceanic structures from satellite images. The location of oceanic structures is very relevant for the study of marine circulation, atmosphere-ocean exchange, and it also has an economic importance. Soft computing technologies such as fuzzy logic have been considered for solving the problem. The proposed system will important to protect people, area near ocean from eddies, upwelling or wakes

\section{Chapters in Books:}

\section{References}

11] Patricia Melin, and Oscar Castillo, "Type-2 Fuzzy Logic in Image Analysis and Pattern Recognition" Advances in Type-2 Fuzzy Sets and Systems, Studies in Fuzziness and Soft Computing 301, DOI:10.1007/978-1-4614-6666-6_12, Springer Science+Business Media, New York 2013

\section{Proceedings Papers:}

[2] Jose A. Piedra-Fernandez, Gloria Ortega, James Z. Wang, and Manuel Canton-Garbin, "Fuzzy Content-Based Image Retrieval for Oceanic Sensing” IEEE Transactions On Geo-Science And Remote Sensing, VOL. 52, NO. 9, September 2014

[3] Patricia Melin, Claudia I. Gonzalez, Juan R. Castro, Olivia Mendoza, and Oscar Castillo, "Edge Detection Method for Image Processing based on Generalized Type-2 Fuzzy Logic" IEEE Transactions on Fuzzy Systems,TFS-2013-0313.R2

[4] Maowen Nie, and Woei Wan Tan, "Analytical Structure and Characteristics of Symmetric Karnik-Mendel Type-Reduced Interval Type-2 Fuzzy PI and PD Controllers" IEEE Transactions on Fuzzy Systems, VOL. 20, NO. 3, June 2012

[5] Zhengyu Huang, Kwang Y. Lee, and Robert M. Edwards, "Fuzzy Logic Control Application in Nuclear Power Plant"15th Triennial World Congress, Barcelona, Spain 2002 